Research Paper

\title{
MiR-145 functions as a tumor suppressor in Papillary Thyroid Cancer by inhibiting RAB5C
}

\author{
Wei Zhang1, Wenyue $\mathrm{Ji}^{2}$, Tianshu $\mathrm{Li}^{2}$, Ting $\mathrm{Liu}^{2}$ and Xudong Zhao ${ }^{{ }^{\circledR}}$ \\ 1. Department of Endocrinology, Shengjing Hospital of China Medical University, Shenyang, 110004, China \\ 2. Department of Otolaryngology head and neck surgery, Shengjing Hospital of China Medical University, Shenyang, 110004, China. \\ $\triangle$ Corresponding author: Dr. Xudong Zhao. Department of Otolaryngology head and neck surgery, Shengjing Hospital of China Medical University, No. 36 \\ Sanhao Street, Heping District, Shenyang, 110004, China; Tel.: +86-024-96615-61311; Fax: +86-024-96615-61311; E-mail: zhaoxdent@hotmail.com. \\ (c) The author(s). This is an open access article distributed under the terms of the Creative Commons Attribution License (https://creativecommons.org/licenses/by/4.0/). \\ See http:/ /ivyspring.com/terms for full terms and conditions.
}

Received: 2020.02.09; Accepted: 2020.07.14; Published: 2020.07.25

\begin{abstract}
Papillary thyroid carcinoma (PTC) accounts for the largest proportion of thyroid cancers; and its morbidity rate has dramatically increased in recent decades. However, the pathogenesis mechanisms of PTC are still not clear. This study aimed to reveal that miR-145 acts as an antitumor miRNA in the progression of PTC. In the present study, the expression of miR-145 was analyzed in 57 paired PTC patient samples. The relationship between clinicopathological features and miR-145 expression were also defined. The tumor suppressive function of miR-145 on PTC cell metastasis, proliferation and apoptosis were revealed in vitro. Also, we used dual luciferase reporter assay to define the relationship of miR-145 and RAB5C. RAB5C was reported to participate in cell invasion and cell motility. We found that miR-145 was downregulated in PTCs, which was negatively correlated with PTC progression and metastasis. MiR-145 inhibited PTC migration, proliferation and promoted apoptosis by directly suppresing RAB5C. In conclusion, miR-145 functions as a tumor suppressor in PTC by inhibiting RAB5C. MiR-145 and RAB5C are potential therapeutic targets in therapy of aggressive PTC cases.
\end{abstract}

Key words: miR-145, RAB5C, papillary thyroid carcinoma, PTC

\section{Introduction}

Thyroid carcinoma accounts for the most frequent malignancy of endocrine organs [1]. Papillary thyroid carcinoma (PTC) accounts for almost $80 \%$ of all types of thyroid cancers and its morbidity rate has dramatically increased in recent decades [2,3]. Although PTC patients generally have a good prognosis, a small proportion of PTC patients have an aggressive form and are prone to further developing progressive disease [4,5]. Thus, it is necessary to reveal the cancer progression mechanism and find inhibitory factors.

MicroRNAs are small noncoding RNAs of 18-22 nucleotides which exert their regulatory functions through inhibition of translation or mRNA degradation [6-9]. MicroRNAs can directly bind to the complementary 3'-untranslated region (3'-UTR) of mRNAs, which results in the inhibition of protein translation [10]. MiR-145 was reported as a tumor suppressor of many solid tumors, such as pancreatic cancer [11], oral squamous cell cancer [12], laryngeal cancer [13], breast cancer [14-18], colorectal cancer [19-21] and gastric cancer [22-24]. However, the function and mechanisms of miR-145 in PTC is still not clear.

Rab5 protein is a type of guanosine triphosphatase, which is related to endosomal classification and participates in the fusion of endosomal membrane [25]. In humans, RAB5 family includes three isoforms (A, B, and C) sharing more than $90 \%$ of sequence identity. The three isoforms may have different functions [26-28]. The RAB5C isoform was reported to participate in cell invasion and cell motility. Chen et al. found that RAB5C modulated Rac-mediated cell motility [29]. Also, RAB5C was reported to regulate cell cohesion [30]. Furthermore, RAB5C was shown to promote ovarian cancer and play important role in drug-resistance of ovarian cancer treatment [31]. Tan et al. found that RAB5C was an important regulator in acute lymphoblastic leukemia [32]. Also, Onodera et al. revealed 
that RAB5C promoted breast cancer invasion by AMAP1-PRKD2 complex [33]. At present, the function of RAB5C in PTC remains largely not clear. The relationship of RAB5C and microRNAs was also reported by Tan et al. RAB5C was established as a target of miR-509 in the progression of acute lymphoblastic leukemia [32].

In current study, we reveal that miR-145 represses PTC progression by suppressing RAB5C for the first time.

\section{Materials and Methods}

\section{Patient tissue samples and PTC cell line}

Fifty-seven paired human PTC samples and adjacent healthy thyroid samples were acquired from patients in Shengjing Hospital of China Medical University between March 2015 and October 2016 (Supplementary Table 1). We acquired prior informed consent from all patients. The study protocol was approved by Shengjing Hospital Ethics Committee. Samples were obtained during surgical resections, immediately frozen in liquid nitrogen and stored at $-80^{\circ} \mathrm{C}$ until use. The human papillary thyroid cancer cell line, BCPAP and K1, were obtained from the cell bank of Biochemistry and Cell Biology Institute of Chinese Academy of Sciences (Shanghai, China).

\section{Quantitative reverse-transcription polymerase chain reaction (qRT-PCR)}

TRIzol reagent (Invitrogen, USA) was used to isolate total RNA from cells and clinical tissues. MiR-145 qRT-PCR assays were conducted by using an AmirVana $^{\mathrm{TM}}$ miRNA detection kit (Ambion, USA) and a Real-Time PCR system purchased from Applied biosystems, USA. Also, we used the $2-\Delta \Delta \mathrm{CT}$ method to determine the relative miR-145 expression. We also used U6 as an endogen control. The reactions were performed following cycling protocol: 1 cycle at $95^{\circ} \mathrm{C}$ for $2 \mathrm{~min}$ followed by 40 cycles of $95^{\circ} \mathrm{C}$ for $8 \mathrm{~s}$ and $60^{\circ} \mathrm{C}$ for $40 \mathrm{~s}$.

CDNA synthesis was performed with the following primer:

- 5'-CTCAACTGGTGTCGTGGAGTCGGCAATT CAGTTGAGCTTTGGGA-3'.

The qRT-PCR primers for U6 were as follows:

- Forward, 5'-CTCGCTTCGGCAGCACATAT ACT-3';

- Reverse, 5' - ACGCTTCACGAATTTGCGTGTC. follows:

The qRT-PCR primers for miR-145 were as

- Forward, 5'-TCGGCAGGGTCCAGUUUUCCC AGG-3';

- Reverse, 5'-CAGTGCGTGTCGTGGAGT -3'.

\section{Western blotting}

Tissues or BCPAP and K1 cells were firstly lysed by RIPA buffer (Beyotime, Shanghai, China) with 10 nM PMSF for 60 minutes and then extract total protein. Sodium dodecyl sulfate polyacrylamide gel electrophoresis (SDS-PAGE) was used to fractionate protein samples, and then proteins were transferred to PVDF membrane. The PVDF membrane was then blocked by $5 \%$ non-fat milk. After that, Rab5c or $\beta$-actin primary antibody (1: 1000, BD Technology, USA) were incubated with the PVDF membrane at $4^{\circ} \mathrm{C}$ overnight. After incubating with secondary antibody, UVP Image System (BD, USA) was used to detect the immunoreactive protein bands. Relative protein levels were quantified by Image J software.

\section{Cell culture and transfection}

RPMI 1640 medium (Gibco, Grand Island, NY, USA) with $10 \%$ fetal bovine serum (FBS) purchased from Gibco, USA was used to culture BCPAP cells. Also, DMEM medium (Gibco, Grand Island, NY, USA) with $10 \%$ fetal bovine serum (FBS) purchased from Gibco, USA was used to culture K1 cells. Cells were cultured in their medium supplied with $10 \%$ FBS (fetal bovine serum) at $37^{\circ} \mathrm{C}$ in a humidified incubator containing 5\% CO2. Two miR-145 mimics and a negative control were obteined from Cyagen Biosciences Inc. (China). RAB5C-specific siRNA and overexpression vector were also obtained from Cyagen Biosciences Inc. (China). Lipofectamine $2000^{\mathrm{TM}}$ purchased from Invitrogen was used for cell transfection. Cells were transfected with miR-145 mimics or controls using Lipofectamine $2000^{\mathrm{TM}}$ according to the manufacturer's protocol. The miR145 mimics transfection concentration was $50 \mathrm{nM}$.

\section{Cell proliferation assay}

A Cell Counting Kit-8 (CCK-8) purchased from Beyotime was used to conduct the cell proliferation assay. Before inoculation, the first choice was the appliance, which used a 96-well plate on which BCPAP or K1 cells were inoculated at a density of 200 cells/Wells. Incubation was conducted after inoculation wherein $10 \mu \mathrm{l}$ of CCK-8 reagent was added separately to $24,48,72$ and $96 \mathrm{~h}$ incubation wells. A microplate reader purchased from Thermo Fisher (China) was used to measure the $450 \mathrm{~nm}$ absorbance.

\section{Cell migration assay}

The migration of BCPAP or K1 cells was evaluated using Transwell assays (BD Biosciences, USA). After $24 \mathrm{~h}$ transfection, we harvested and resuspended the BCPAP or K1 cells, and then seeded into the upper chambers at $4 \times 10^{4}$ cells/well density. 
Then, $0.5 \mathrm{ml}$ of DMEM or RPMI 1640 medium with $20 \%$ FBS was added to each chamber. The BCPAP or $\mathrm{K} 1$ cells were allowed to migrate for $24 \mathrm{~h}$ at $37^{\circ} \mathrm{C}$. Then, we used $4 \%$ paraformaldehyde to fix the migrated cells on the lower surface for $10 \mathrm{~min}$. After that, crystal violet solution was used to stain the cells for $15 \mathrm{~min}$ at room temperature. Then we used a photomicroscope (BX51; Olympus, Japan) to image the BCPAP or K1 cells on lower surfaces.

\section{Luciferase reporter assay}

BCPAP or K1 cells were cotransfected with RAB5C-3'-UTR-WT or Rab5c-MUT and miR-145 mimic or the negative control, using Lipofectamine 2000. BCPAP or K1 cells were transfected for $48 \mathrm{~h}$. After that, Dual-Luciferase Reporter Assay System (Promega, CA, USA) was used for analysis. A GloMax fluorescence reader (Promega) was used to determine the luciferase activity. All experiments were repeated thrice.

\section{Flow cytometry and quantification of apoptotic cells}

We seeded $0.3 \times 10^{6} \mathrm{BCPAP}$ or $\mathrm{K} 1$ cells each well and cultured overnight. Then we harvested and washed thrice using fluorescence-activated cell sorting buffer. The cells were incubated with Fc blocker at room temperature for $30 \mathrm{~min}$. After that, the BCPAP or $\mathrm{K} 1$ cells were stained by Anti-Annexin $\mathrm{V}$ antibody (Abcam, USA) for $20 \mathrm{~min}$ at room temperature according to the manufacturer's protocol. Then we used an LSR II flow cytometer (BD Biosciences) to run the flow. Also, FlowJo software (Tree Star) was used to analyze the results. BCPAP or $\mathrm{K} 1$ cells treated with $0.1 \mathrm{~mol} / \mathrm{L}$ 5-fluorouracil (5-FU) were used as positive control.

\section{Colorimetric caspase- 3 assay}

We lysed BCPAP or K1 cells and calculated the protein concentrations. One hundred microgram of proteins was treated with $10 \mu \mathrm{L}$ of Ac-DEVD-pNA (Abcam, USA) and incubated at $37^{\circ} \mathrm{C}$ for $2 \mathrm{~h}$. Then we used a microplate reader (Bio-Tek Instruments) to determine the $405 \mathrm{~nm}$ absorbance.

\section{Statistical analysis}

All experiments were repeated for at least three times. We used the mean \pm SD to summarize all the data. Also, we used SPSS 17.0 (SPSS Inc., USA) for all the statistical analyses. Firstly, all data were assessed by normal distribution test. And all the normally distributed data were analyzed by Student's $t$-test. Ratio results were log-transformed prior to Student's $t$-tests. $P<0.05$ was considered statistically significant in all analyses.

\section{Results}

\section{Downregulation of miR-145 in human PTC tissues}

QRT-PCR were used to analyze the miR-145 expression in PTC and adjoining non-tumor tissue samples. We found that 46 PTC specimens had reduced miR-145 expression when comparing adjoining non-malignant thyroid tissue samples (Figure 1A) .Compared with adjoining normal tissues, PTC tissues exhibited less of the expression of miR145. The mean relative expression level of miR-145 in total non-malignant thyroid tissue was $10.01 \pm 3.56$ and in the whole malignant sample $3.56 \pm 1.46(\mathrm{P}=$ 0.005). Therefore, compared to adjacent nonmalignant thyroid tissue, PTC cases exhibited about 2.81-twice lower level of miR-145 expression (Figure 1B).

\section{MiR-145 act as a suppresser in PTC progression and metastasis}

The clinical significance of miR-145 in human PTC is still unclear. The clinicopathological parameters in patient records of the 57 patients were reviewed. Tumor size, age, extrathyroidal invasion, sex, cervical lymph node metastasis and multicentricity were analyzed for associations with the expression of miR-145 (Table 1). There were no significant associations between the expression of miR-145 and age, sex, tumor size, multicentricity, extrathyroidal invasion. But the expression of miR145 was significantly upregulated in patients in the early-TNM stage and without cervical lymph node metastasis. Collectively, these results suggested that miR-145 act as a suppressor in human PTC progression and metastasis.

\section{Enhanced miR-145 expression suppresses proliferation and migration, and promotes apoptosis of BCPAP and $K 1$ cells in vitro}

We used MTT assays to determine the influence of miR-145 overexpression on the proliferation of BCPAP and K1 cells. We transiently transfected BCPAP and K1 cells with miR-145 mimics or negative control (Figure 2A). QPCR was used to identify the expression of miR-145 in each (BCPAP cells: $10.91 \pm$ 0.17 and $9.87 \pm 0.25$ vs. $3.61 \pm 0.08$ [control], $P<0.001$; K1 cells: $10.31 \pm 0.22$ and $8.94 \pm 0.41$ vs. $3.48 \pm 0.02$ [control], $P<0.001)$. MTT assays demonstrated that transfection with miR-145 mimic suppressed the growth of BCPAP and $\mathrm{K} 1$ cells comparing with the negative control in a time-dependent manner. Decline with time was found in the cell rate of increase in cell number of the miR-145-overexpression cells (Figure $2 \mathrm{~B})$. To determine the migration potential of BCPAP 
and $\mathrm{K} 1$ cells overexpressing miR-145, we used Transwell assays. MiR-145 overexpression resulted in decreased BCPAP and $\mathrm{K} 1$ cell migration comparing with negative control (Figure 2C). Next, Annexin-V staining was used to determine the influence of miR-145 on PTC apoptosis. Overexpression of miR-145 significantly enhanced BCPAP and K1 cell apoptosis (Figure 2D). Also, similar results were obtained using colorimetric caspase 3 assay to evaluate BCPAP and K1 cell apoptosis (Figure 2E). Taken together, these data clearly indicated that miR-145 can inhibit PTC cell migration and proliferation, also promotes apoptosis.

\section{MiR-145 can directly inhibit RAB5C expression in PTC}

Bioinformatics analysis (miRWalk, TargetScan, and PicTar) predicted that RAB5C is a direct target of miR-145. We determined the relationship between miR-145 and RAB5C in K1 and BCPAP cells. MiR-145 mimic-mediated miR-145 overexpression was associated with a significant decrease in RAB5C expression comparing with the negative control. RAB5C relative expression was suppressed $62.8 \%$ in BCPAP cells and $66.2 \%$ in $\mathrm{K} 1$ cells separately by miR145 overexpression (Figure 3A). We determined the association of miR-145 and RAB5C by using luciferase reporter assays. Firstly, we constructed luciferase reporter plasmids which contains a mutant or the wild-type 3'-UTR of RAB5C (Figure 3B). We found significant decrease of luciferase activity of wild-type RAB5C 3'-UTR reporter upon cotransfection with miR-145 mimic, whereas that of the mutant 3'-UTR reporter was unaffected by cotransfection with

A
miR-145 mimic (Figure 3C). Collectively, these suggested that miR-145 can directly inhibit RAB5C in PTC.

Table 1. Correlation of miR-145 expression with clinicopathological factors of PTC patients

\begin{tabular}{|c|c|c|c|}
\hline Parameters & Patients & miR-145 relative expression & $P$ \\
\hline Total gender & 57 & & 0.641 \\
\hline Male & $14(24.6)$ & $3.71 \pm 1.54$ & \\
\hline Female & $43(75.4)$ & $3.48 \pm 1.43$ & \\
\hline Age (Y) & & & 0.213 \\
\hline$\geq 45$ & $33(57.9)$ & $3.37 \pm 1.57$ & \\
\hline$<45$ & $24(42.1)$ & $3.83 \pm 1.34$ & \\
\hline Tumor size, cm & & & 0.097 \\
\hline$\geq 2$ & $25(43.9)$ & $3.29 \pm 1.75$ & \\
\hline$<2$ & $32(56.1)$ & $3.85 \pm 1.09$ & \\
\hline Extrathyroidal invasion & & & 0.116 \\
\hline Yes & $13(22.8)$ & $3.38 \pm 2.02$ & \\
\hline No & $44(77.2)$ & $3.95 \pm 1.17$ & \\
\hline Multicentricity & & & 0.437 \\
\hline Yes & 18 (31.6) & $3.40 \pm 1.24$ & \\
\hline No & $39(68.4)$ & $3.89 \pm 1.57$ & \\
\hline Cervical metastasis status & & & 0.022 \\
\hline $\mathrm{N}+$ & $23(45.6)$ & $2.87 \pm 1.60$ & \\
\hline N- & $34(54.4)$ & $4.54 \pm 1.37$ & \\
\hline TNM stages & & & 0.041 \\
\hline $\mathrm{I} / \mathrm{II}$ & $31(54.4)$ & $4.21 \pm 1.26$ & \\
\hline III/IV & $26(45.6)$ & $2.71 \pm 1.71$ & \\
\hline
\end{tabular}

Note: Values in parentheses represent percentages.

\section{Forced RAB5C overexpression restores the inhibitory effects of miR-145}

In order to explore the inhibitory effects of miR-145 targeting RAB5C in PTC, a RAB5C overexpression vector was transfected into BCPAP cells and K1 cells with miR-145 overexpression (Figure 4A) to restore RAB5C expression (BCPAP cells: $49.88 \pm 4.10$ vs. $29.75 \pm 2.41$ transfection with

\section{B}
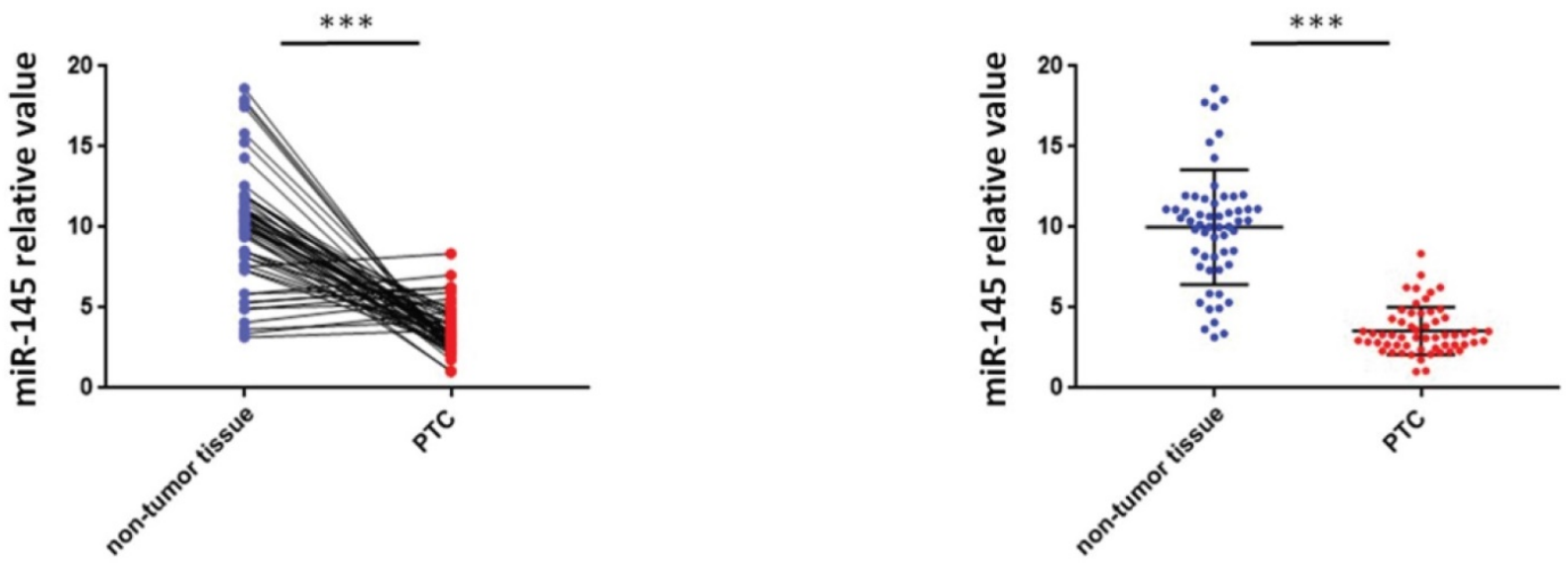

Figure 1. Aberrant expression of miR-145 in PTC tissues. (A) Quantification of the process of reverse transcription polymerase reaction ( $\mathrm{QRT}-\mathrm{PCR}$ ) analysis of miR-145 expression in 57 paired PTC and adjacent non-tumor tissues. We found that $46 / 57$ (80.7\%) of PTC specimens had reduced miR-145 expression compared to adjacent non-malignant thyroid tissue (log-transformed of data was used prior to paired $t$-test analysis; $P=0.002$ ). Each set of experiments was performed three times. The repetition of a creature is represented by a dot. (B) qRT-PCR analysis of miR-145 expression in PTC and adjacent normal tissue samples. Statistical analysis is carried out by t-test method, and the analysis is performed after logarithmic transformation. Each set of experiments was performed three times. The repetition of a creature is represented by a dot. 
mimic alone, $P=0.042 ; \mathrm{K} 1$ cells: $55.52 \pm 5.03$ vs. $20.33 \pm$ 3.17 transfection with mimic alone, $P=0.036$ ). We observed a time-dependent increased proliferation in BCPAP cells with RAB5C rescue, comparing with miR-145 overexpression only $(6.3 \pm 0.3,16.7 \pm 2.3,31.2$ \pm 3.8 , and $42.6 \pm 4.9 \%$ at $24 \mathrm{~h}, 48 \mathrm{~h}, 72 \mathrm{~h}$, and $96 \mathrm{~h}$, respectively, $P=0.021)$; Also, the rescue effect in $\mathrm{K} 1$ cells was also observed $(4.5 \pm 0.5,8.2 \pm 1.9,18.4 \pm 3.1$, and $31.7 \pm 3.8 \%$ at $24 \mathrm{~h}, 48 \mathrm{~h}, 72 \mathrm{~h}$, and $96 \mathrm{~h}$,
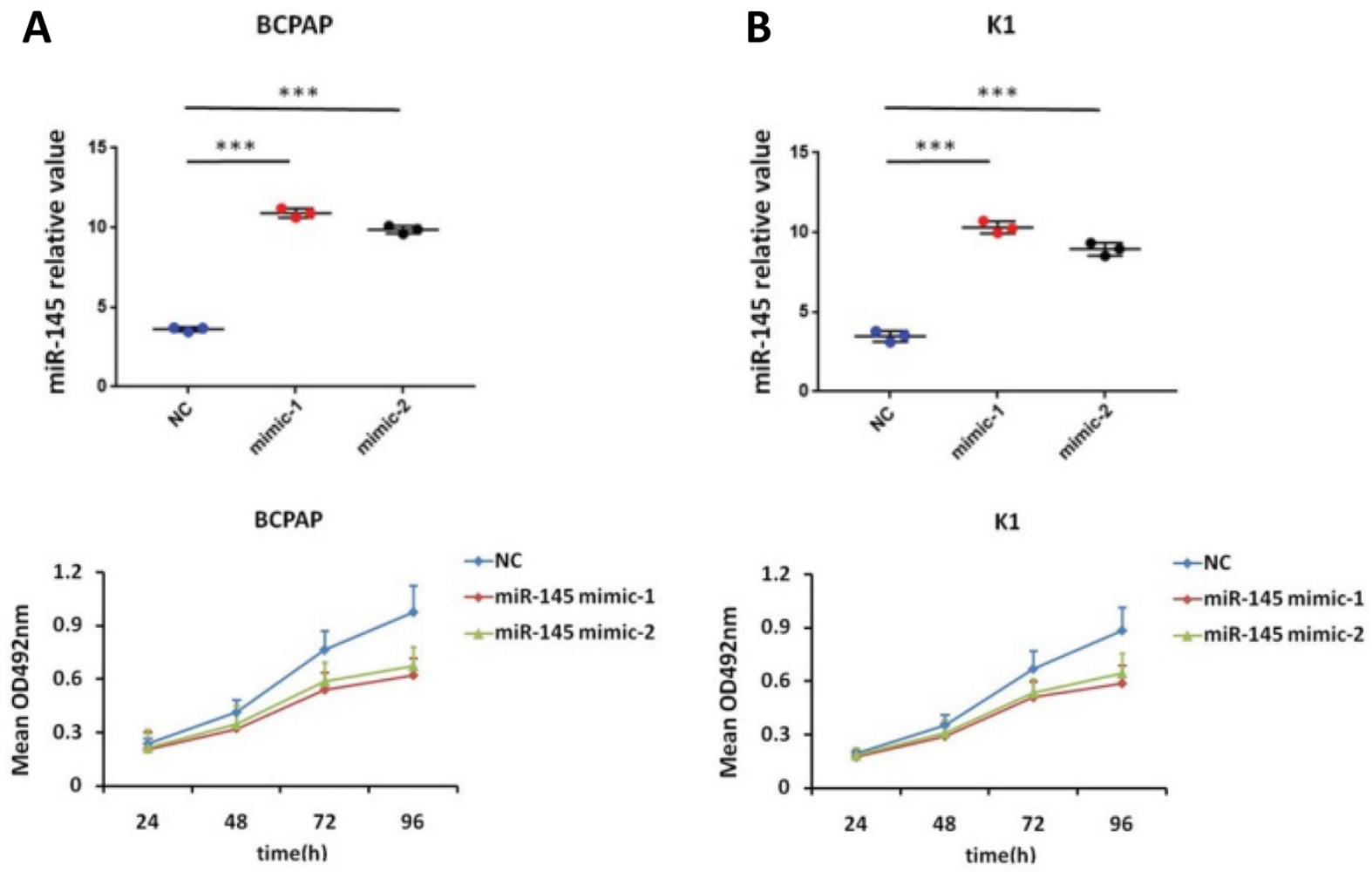

C

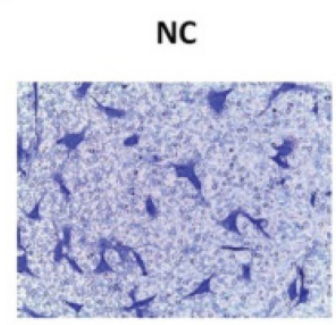

BCPAP

miR-145 mimic-1 miR-145 mimic-2
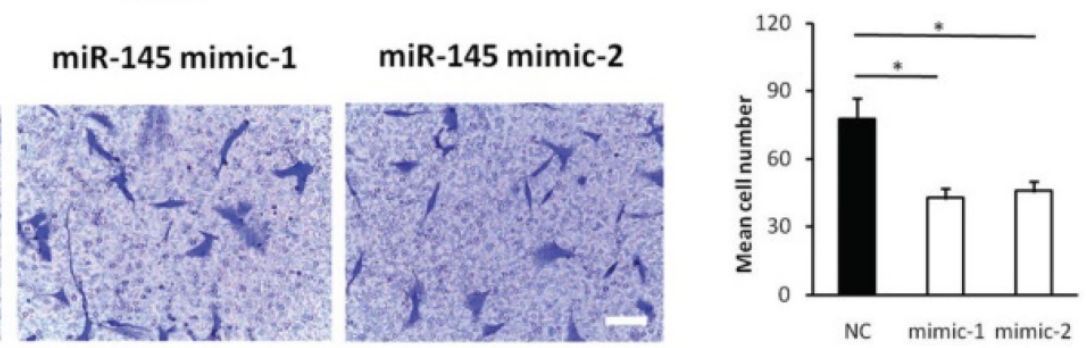

K1

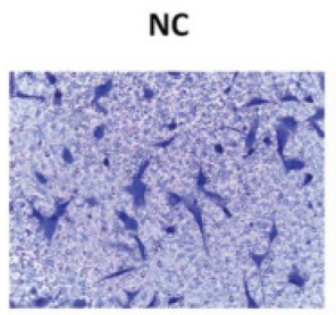

miR-145 mimic-1
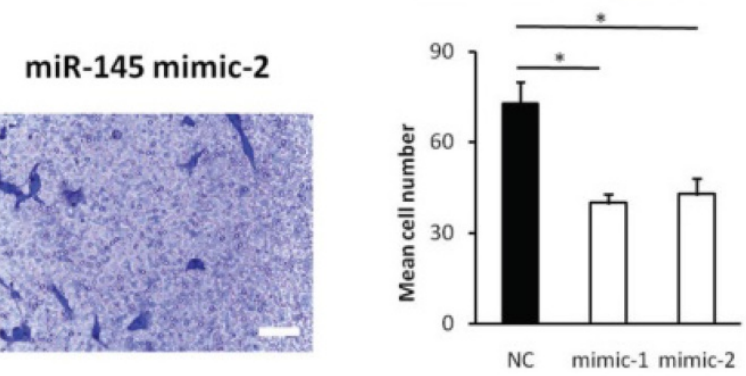
D

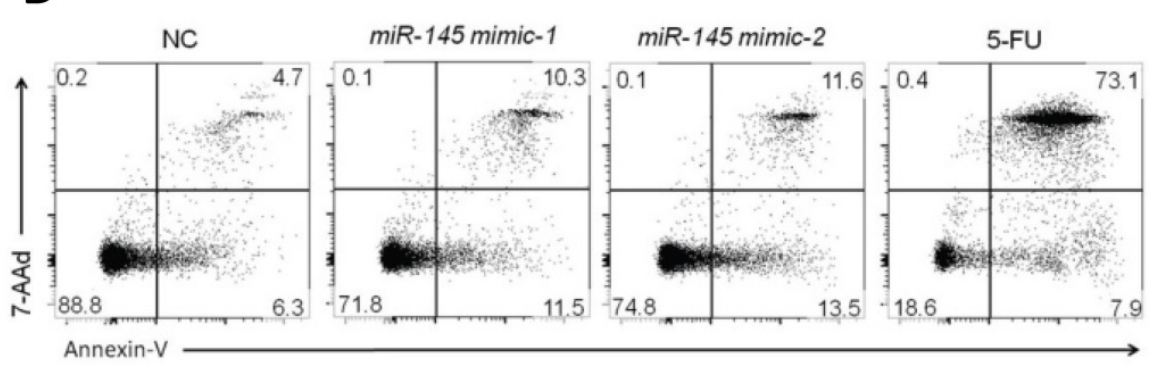

K1

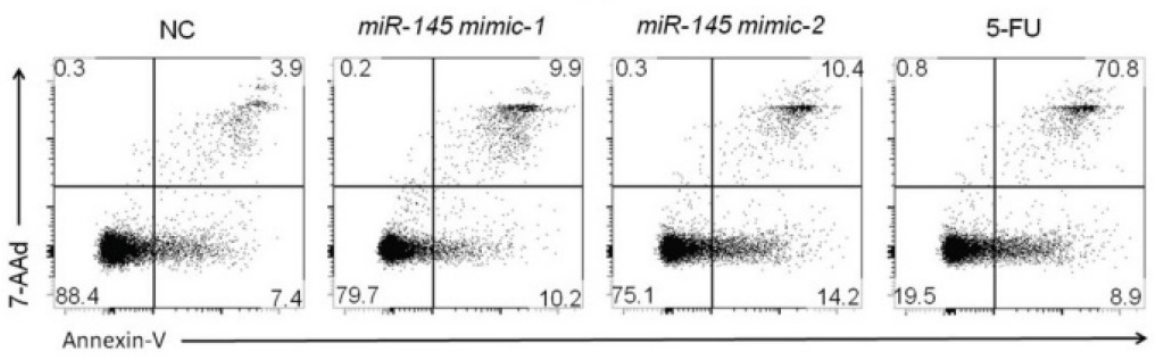

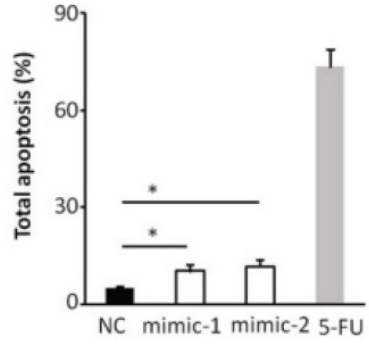

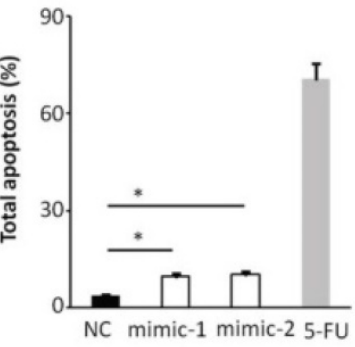

$\mathbf{E}$

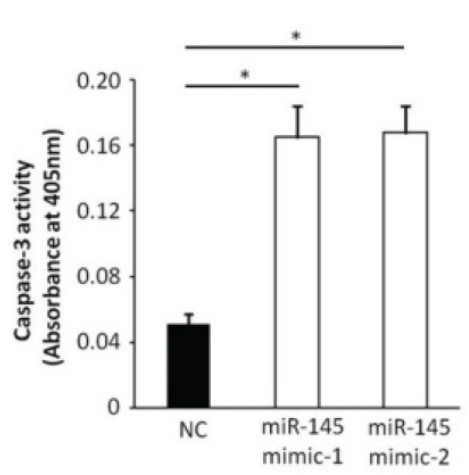

K1

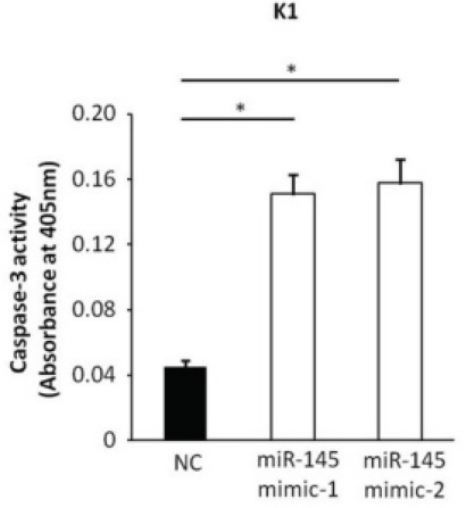

Figure 2. Effect of miR-145 on PTC cell proliferation, migration, and apoptosis. (A) BCPAP and K1 cells were transiently transfected with two mimics of miR-145 or negative control $(P<0.001)$. (B) Cell survival rates determined by MTT assays in miR-145-overexpressed and control BCPAP and K1 cells. (C) Representative photographs of Transwell aggressive attacks on miR-145 overexpression and negative effects on BCPAP and K1 cells (upper) and their quantitative analysis (bottom). Scale bar, 50 mm. (*represents $P<0.05$ ). (D) Representative photographs and proportion of apoptosis of BCPAP and K1 cells with miR-145-overexpression or negative control (NC) and positive control (treated with 5 -FU) $(*$ represents $P<0.05$ ). (E) Caspase-3 activity in BCPAP and K1 cells transfected with the mimics of miR-145 or negative control $(*$ represents $P<$ 0.05). Each set of experiments was performed three times. The repetition of a creature is represented by a dot.

\section{Discussion}

In endocrine system, thyroid cancer is the most common, and the incidence has increased in past decades. PTC accounts for the most common portion in all the thyroid cancer [34]. Most PTC patients can be effectively treated with surgery; however, $10 \%-$ $15 \%$ of patients with PTC have poor prognosis [35]. Therefore, the identification of potential targets which can inhibit the progression in PTC is urgently needed.

The miR-145 gene is found located on chromosome 5 (5q32-33) [36]. MiR-145 reportedly acts as an inhibitor in numerous solid malignancies. Azmi et al. highlighted miR-145 as a tumor suppressor in pancreas [11]. Rodrigues et al. reported that miR-145 inhibits oral squamous cell carcinoma progression
[12]. Devlin et al. revealed that miR-145 has anticancer effects in breast cancer [15]. Hu et al. revealed that miR-145 inhibited the progression of gastric cancer [22]. Moreover, several researches have revealed that miR-145 is a novel biomarker of various solid malignancies $[19,21,24]$. In this study, we found that the expression of miR-145 was decreased in 46 out of 57 PTC tissues evaluated. Eighty percent of the PTC showed decreased miR-145 expression. That means the expression of miR-145 was generally decreased in PTC. After that, in order to determine the clinical significance of miR-145, we analyzed potential correlations between miR-145 and clinicopathological factors. We found that lower miR-145 expression associated with advanced stages and the presence of cervical metastasis. So we believe that the lower 
miR-145 expression may promote cervical metastasis in clinical practice. Also, patients with large tumor size showed lowered miR-145 expression, but this finding is not statistically significant.

After that, BCPAP and K1 cells were used to reveal the effect and mechanism of aberrant miR-145 expression in PTC. The online tools were used to predict the targets of miR-145. Then, RAB5C was identified as a potential target of miR-145. RAB5C is a type of guanosine triphosphatase which participate in endosomal membrane fusion reactions and can regulate sorting endosome [25]. Jin et al. reported aberrant expression of RAB5C in ovarian cancer [31]. Moreover, Onodera et al. revealed that RAB5C promoted invasion of breast cancer cells [33]. In our present study, we increased miR-145 expression in cell lines. And we found the proliferation and invasion of cells were inhibited and apoptosis increased significantly. These results confirmed miR-145 can inhibit the progression of PTC, the same as previous conclusions in clinical data. Also, BCPAP and $\mathrm{K} 1$ cells were used to reveal the correlation between miR-145 and RAB5C. MiR-145 overexpression was correlated with significant inhibition of RAB5C expression. Luciferase reporter assays were used to show that miR-145 can directly bind to the 3'-UTR of RAB5C, affecting the RAB5C expression. These results indicated that $\mathrm{RAB} 5 \mathrm{C}$ is target of

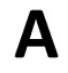

BCPAP

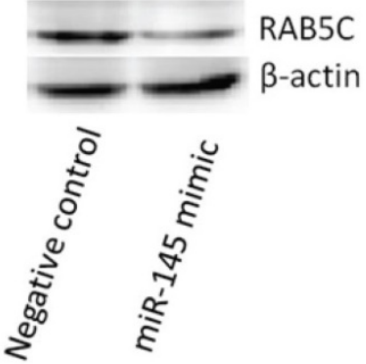

K1

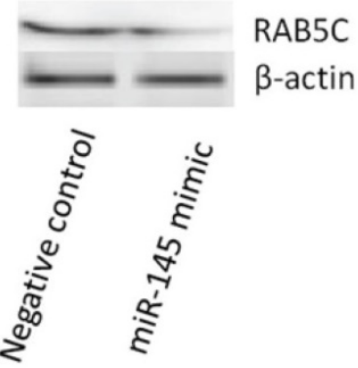

B

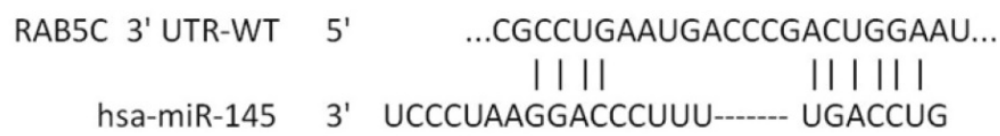

RAB5C 3' UTR-Mutant $\quad 5^{\prime} \quad$...CGGGACAAUGACCCGUGACCUGU...

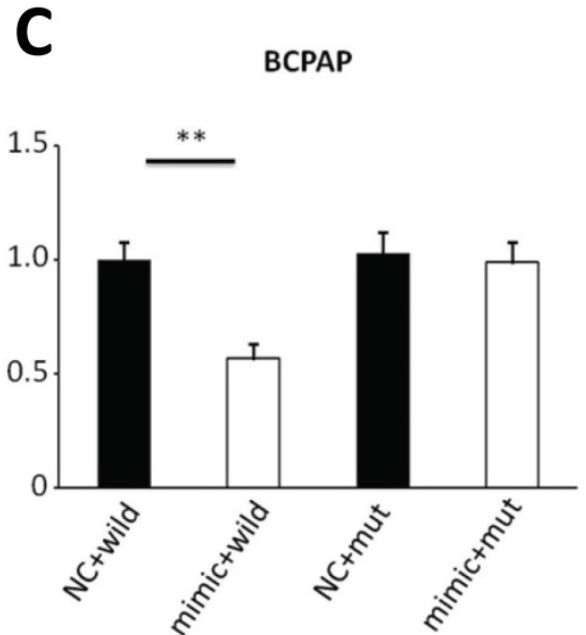

K1

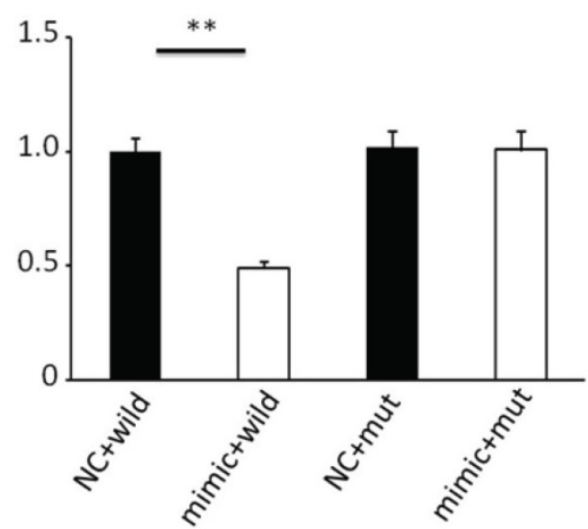

Figure 3. RAB5C is a direct target of miR-145 in PTC. (A) Analysis of representative western blot and RAB5C proteins by miR-145 simulation or negative comparison analysis of BCPAP and KI cells $(P=0.031$ and $P=0.040)$. $\beta$-actin was used as an endogen control. (B) miR-145 straight from the shoulder interacted with the $3^{\prime}-U T R s$ of RAB5C. (C) After co-transfection with miR-145 mimic, the relative luciferase activity of the reporter plasmid containing the uncultivated-type RAB5C 3'-UTR was significantly reduced. But the miR-145 level change did not produce an effect on the luciferase activity of the reporter plasmid with the RAB5C mutant 3'-UTRs. These data are the mean \pm SD of the results of three trials that do not interfere with each other. After the logarithmic transformation of the data by two-way analysis of variance, it is statistically organized and analyzed. $* * P=0.022$ and 0.015 for BCPAP and K1 cells, respectively. Dots represent experimental repetitions. 
miR-145 in PTC. Moreover, we found that overexpression of RAB5C reversed the effects of miR-145 overexpression on cell migration and proliferation, and apoptosis. These results revealed that miR-145 can inhibit the PTC progression via RAB5C. But in our results we found that RAB5C reversed the effects of miR-145 overexpression partially, not completely. So we speculated there were other downstream targets of miR-145 in PTC. Our further study will focus on this.
A

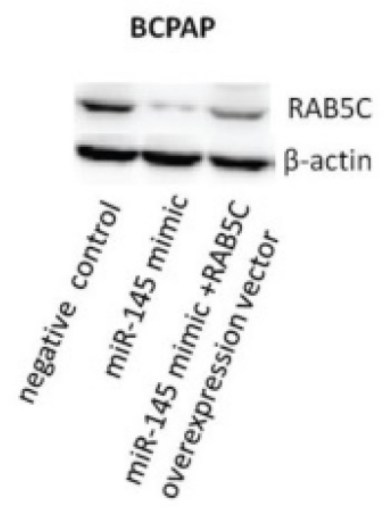

B

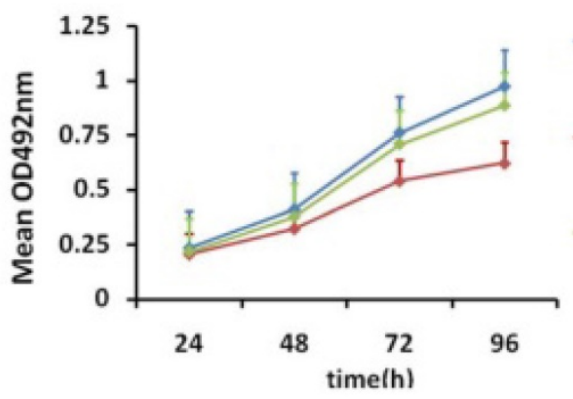

$\rightarrow$ NC

$\rightarrow$ miR-145 mimic

-miR-145 +RAB5C overexpression
K1

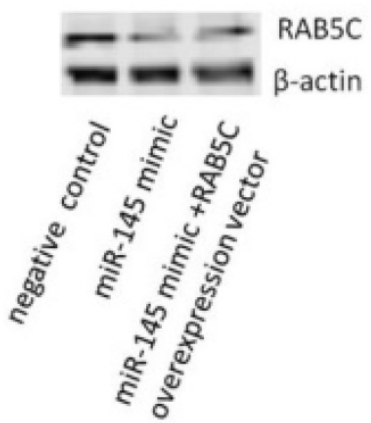

C

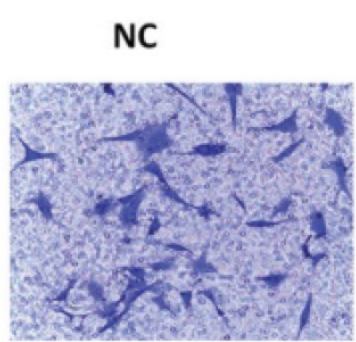

miR-145 overexpression

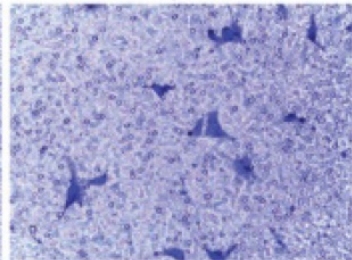

miR-145+RAB5C overexpression

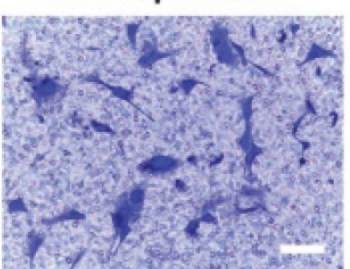

K1
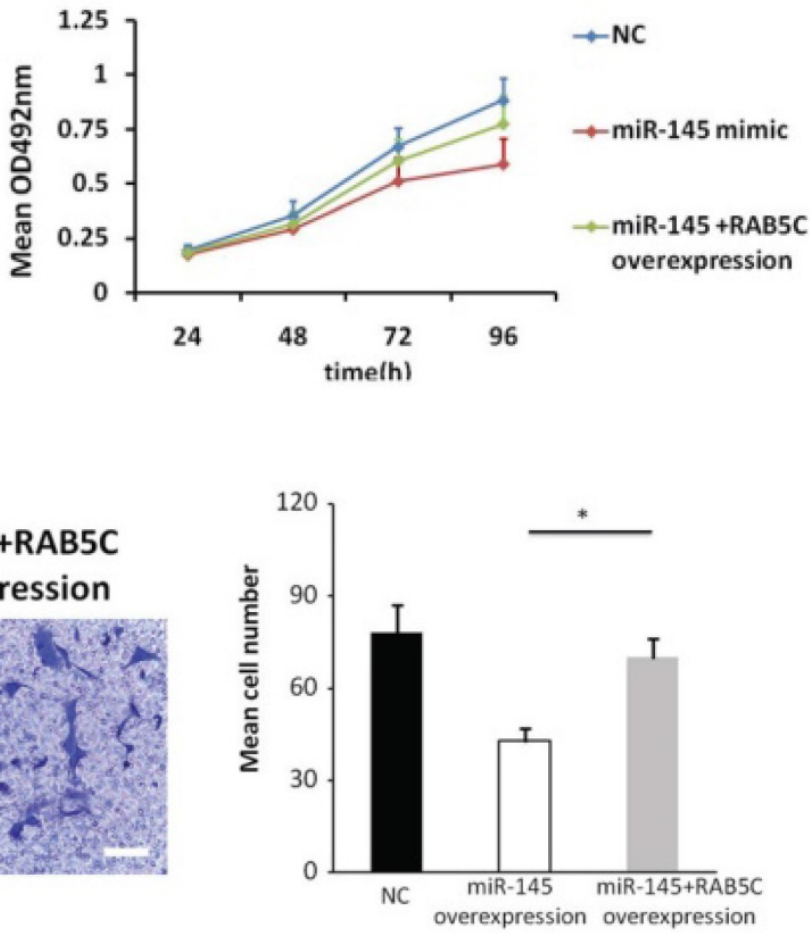

K1

NC

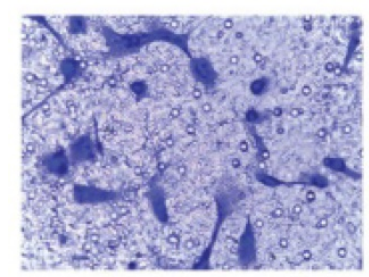

miR-145 overexpression

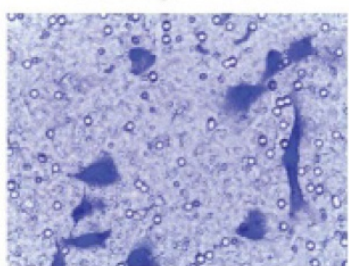

miR-145+RAB5C overexpression

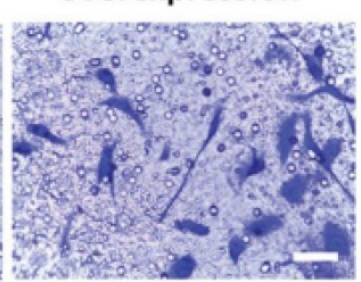


D

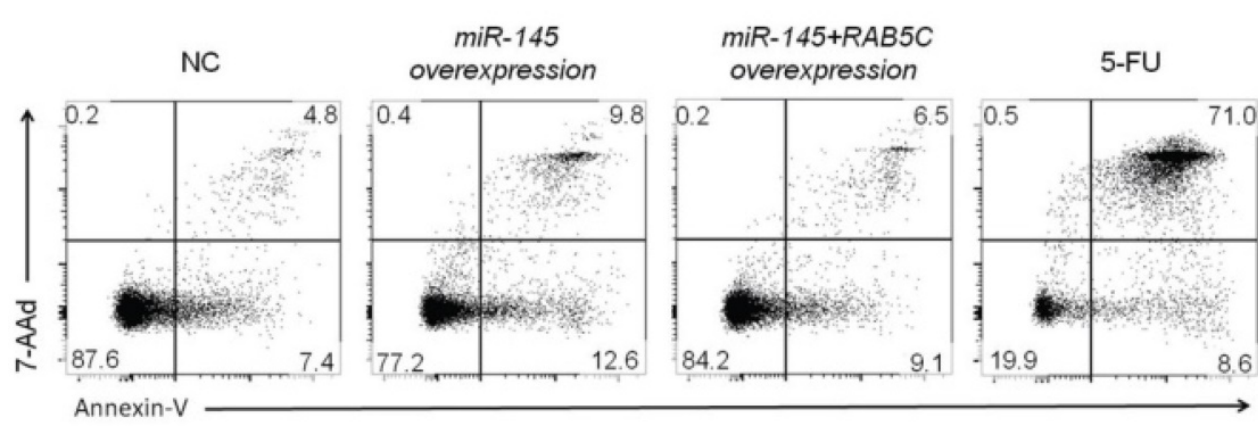

K1
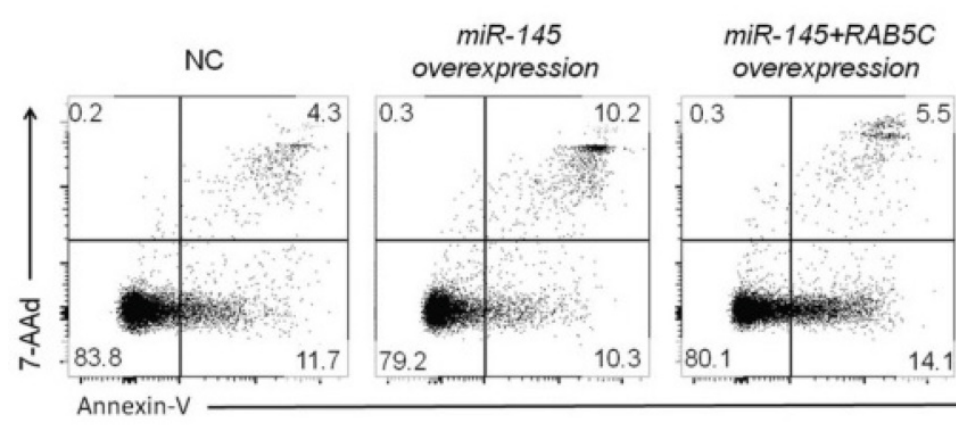

14.1

E

BCPAP

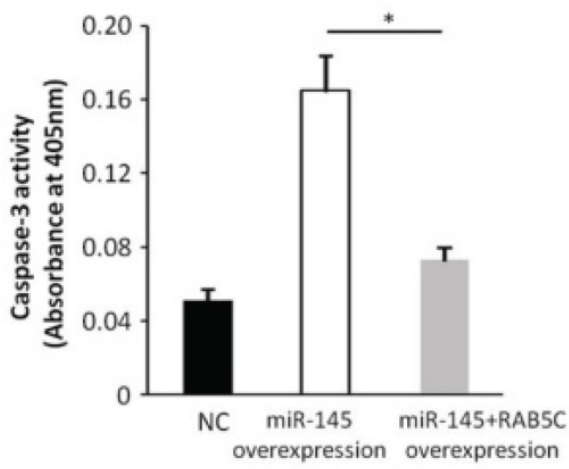

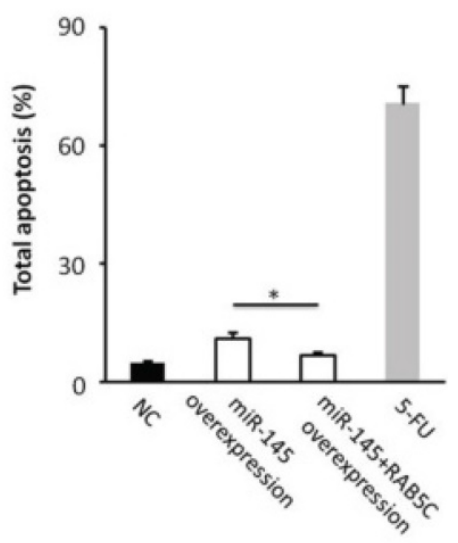

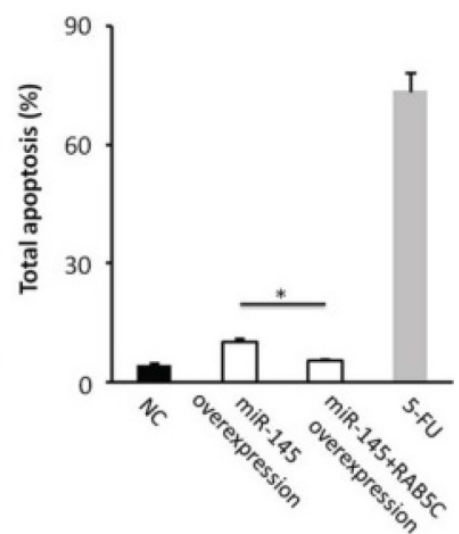

K1

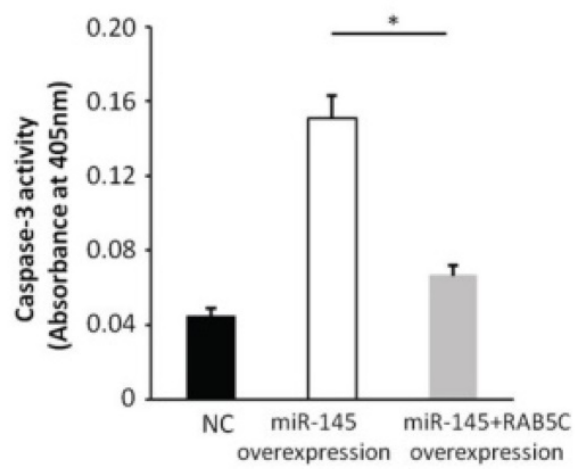

Figure 4. RAB5C overexpression restores the inhibitory effects of miR-145. (A) Typical western blot findings delivery that transfection of RAB5C-overexpression carrier comtrastto restores the depression of RAB5C after transfection with miR-145 mimic. (B) Value added rates of miR-145-overexpression BCPAP and K1 cells at different time points after RAB5C overexpression. (C) Typical pictures (upper) of Transwell aggressive attacks of miR-145-overexpressing BCPAP and K1 cells with or without RAB5C overexpression and analysis of specific quantities (bottom) Scale bar, $50 \mu \mathrm{m}$. $(P=0.035$ and $P=0.023)$. (D) Typical pictures showing the ratio of BCPAP and K1 cells in apoptosis after transfection with miR-145 mimic with and without RAB5C overexpression $(P=0.036$ and 0.031$)$. (E) Caspase- 3 doings of $B C P A P$ and $K 1$ cells transfection with miR-145 mimic have and without RAB5C over-deliverance $(P=0.029$ and 0.018$)$. NC: negative control.

Our study had some limitations. Namely, further research of tumorigenicity assays on nude mice is needed to show the role of miR-145 and RAB5C in PTC progression.

Conclusively, our study revealed that aberrant miR-145 expression affects PTC progression: miR-145 inhibition causes RAB5C overexpression and cancer progression. Therefore, miR-145 and RAB5C could be potential therapeutic targets in the therapy of aggressive PTC cases.

\section{Supplementary Material}

Supplementary table.

http://www.medsci.org/v17p1992s1.pdf 


\section{Acknowledgements}

This study was supported by the National Natural Science Foundation of China (No. 81302364) and Education Department of Liaoning Provincial (No. QN2019012). These two funding played an important role in experimental conduction, data collection, and data analysis.

\section{Ethics approval and consent to participate}

The Ethics Committee of the Shengjing Hospital of China Medical University approved this study (2014PS18K). All patients involved in the study provided written informed consent.

\section{Competing Interests}

The authors have declared that no competing interest exists.

\section{References}

1. Xia Q, Dong S, Bian PD, Wang J, Li CJ. Effects of endocrine therapy on the prognosis of elderly patients after surgery for papillary thyroid carcinoma. European archives of oto-rhino-laryngology : official journal of the European Federation of Oto-Rhino-Laryngological Societies. 2016;273(4):1037-43.

2. Chiappetta G, Valentino T, Vitiello M, Pasquinelli R, Monaco M, Palma G, et al. PATZ1 acts as a tumor suppressor in thyroid cancer via targeting p53-dependent genes involved in EMT and cell migration. Oncotarget. 2015;6(7):5310-23.

3. Peng XG, Chen ZF, Zhang KJ, Wang PG, Liu ZM, Chen ZJ, et al. VEGF Trapon inhibits tumor growth in papillary thyroid carcinoma. European review for medical and pharmacological sciences. 2015;19(2):235-40.

4. DeSantis CE, Lin CC, Mariotto AB, Siegel RL, Stein KD, Kramer JL, et al. Cancer treatment and survivorship statistics, 2014. CA: a cancer journal for clinicians. 2014;64(4):252-71.

5. McCaffrey JC. Aerodigestive tract invasion by well-differentiated thyroid carcinoma: diagnosis, management, prognosis, and biology. The Laryngoscope. 2006;116(1):1-11.

6. Ambros V. The functions of animal microRNAs. Nature. 2004;431(7006):350-5.

7. Bartel DP. MicroRNAs: genomics, biogenesis, mechanism, and function. Cell. 2004;116(2):281-97.

8. Bartel DP. MicroRNAs: target recognition and regulatory functions. Cell. 2009;136(2):215-33.

9. Kosik KS. MicroRNAs and cellular phenotypy. Cell. 2010;143(1):21-6.

10. Jonas S, Izaurralde $\mathrm{E}$. Towards a molecular understanding of microRNA-mediated gene silencing. Nature reviews Genetics. 2015;16(7):421-33.

11. Azmi AS, Li Y, Muqbil I, Aboukameel A, Senapedis W, Baloglu E, et al. Exportin 1 (XPO1) inhibition leads to restoration of tumor suppressor miR-145 and consequent suppression of pancreatic cancer cell proliferation and migration. Oncotarget. 2017;8(47):82144-55.

12. Rodrigues PC, Sawazaki-Calone I, Ervolino de Oliveira C, Soares Macedo CC, Dourado MR, Cervigne NK, et al. Fascin promotes migration and invasion and is a prognostic marker for oral squamous cell carcinoma. Oncotarget. 2017;8(43):74736-54.

13. Karatas OF, Yuceturk B, Suer I, Yilmaz M, Cansiz H, Solak M, et al. Role of miR-145 in human laryngeal squamous cell carcinoma. Head \& neck. 2016;38(2):260-6

14. Bahrami A, Aledavood A, Anvari K, Hassanian SM, Maftouh M, Yaghobzade $\mathrm{A}$, et al. The prognostic and therapeutic application of microRNAs in breast cancer: Tissue and circulating microRNAs. Journal of cellular physiology. 2018;233(2):774-86

15. Devlin KL, Sanford T, Harrison LM, LeBourgeois $\mathrm{P}$, Lashinger LM, Mambo $\mathrm{E}$, et al. Stage-Specific MicroRNAs and Their Role in the Anticancer Effects of Calorie Restriction in a Rat Model of ER-Positive Luminal Breast Cancer. PloS one. 2016;11(7):e0159686.

16. Ghanbari Safari M, Baesi K, Hosseinkhani S. An alternative approach in regulation of expression of a transgene by endogenous miR-145 in carcinoma and normal breast cell lines. Biotechnology and applied biochemistry. 2017;64(2):244-50

17. Han JG, Jiang YD, Zhang CH, Yang YM, Pang D, Song YN, et al. A novel panel of serum miR-21/miR-155/miR-365 as a potential diagnostic biomarker for breast cancer. Annals of surgical treatment and research. 2017;92(2):55-66.

18. Zheng $\mathrm{T}$, Zhang $\mathrm{X}$, Wang $\mathrm{Y}, \mathrm{Yu} \mathrm{X}$. Predicting associations between microRNAs and target genes in breast cancer by bioinformatics analyses. Oncology letters. 2016;12(2):1067-73.
19. Pan C, Yan X, Li H, Huang L, Yin M, Yang Y, et al. Systematic literature review and clinical validation of circulating microRNAs as diagnostic biomarkers for colorectal cancer. Oncotarget. 2017;8(40):68317-28.

20. Shinohara H, Kuranaga Y, Kumazaki M, Sugito N, Yoshikawa Y, Takai T, et al. Regulated Polarization of Tumor-Associated Macrophages by miR-145 via Colorectal Cancer-Derived Extracellular Vesicles. Journal of immunology. 2017;199(4):1505-15.

21. Yang J, Ma D, Fesler A, Zhai H, Leamniramit A, Li W, et al. Expression analysis of microRNA as prognostic biomarkers in colorectal cancer. Oncotarget. 2017;8(32):52403-12.

22. $\mathrm{Hu} \mathrm{CE}, \mathrm{Du} \mathrm{PZ}$, Zhang HD, Huang GJ. Long Noncoding RNA CRNDE Promotes Proliferation of Gastric Cancer Cells by Targeting miR-145. Cellular physiology and biochemistry : international journal of experimental cellular physiology, biochemistry, and pharmacology. 2017;42(1):13-21.

23. Lei C, Du F, Sun L, Li T, Li T, Min Y, et al. miR-143 and miR-145 inhibit gastric cancer cell migration and metastasis by suppressing MYO6. Cell death \& disease. 2017;8(10):e3101.

24. Zhang Y, Wen $\mathrm{X}, \mathrm{Hu} \mathrm{XL}$, Cheng LZ, Yu JY, Wei ZB. Downregulation of miR-145-5p correlates with poor prognosis in gastric cancer. European review for medical and pharmacological sciences. 2016;20(14):3026-30.

25. Woodman PG. Biogenesis of the sorting endosome: the role of Rab5. Traffic. 2000;1(9):695-701.

26. Bucci C, Lutcke A, Steele-Mortimer O, Olkkonen VM, Dupree P, Chiariello M, et al. Co-operative regulation of endocytosis by three Rab5 isoforms. FEBS letters. 1995;366(1):65-71.

27. Chen PI, Kong C, Su X, Stahl PD. Rab5 isoforms differentially regulate the trafficking and degradation of epidermal growth factor receptors. The Journal of biological chemistry. 2009;284(44):30328-38.

28. Wainszelbaum MJ, Proctor BM, Pontow SE, Stahl PD, Barbieri MA. IL4/PGE2 induction of an enlarged early endosomal compartment in mouse macrophages is Rab5-dependent. Experimental cell research. 2006;312(12):2238-51.

29. Chen PI, Schauer K, Kong C, Harding AR, Goud B, Stahl PD. Rab5 isoforms orchestrate a "division of labor" in the endocytic network; Rab5C modulates Rac-mediated cell motility. PloS one. 2014;9(2):e90384.

30. Ulrich F, Krieg M, Schotz EM, Link V, Castanon I, Schnabel V, et al. Wnt11 functions in gastrulation by controlling cell cohesion through Rab5c and E-cadherin. Developmental cell. 2005;9(4):555-64.

31. Jin L, Huo Y, Zheng Z, Jiang X, Deng H, Chen Y, et al. Down-regulation of Ras-related protein Rab 5C-dependent endocytosis and glycolysis in cisplatin-resistant ovarian cancer cell lines. Molecular \& cellular proteomics : MCP. 2014;13(11):3138-51.

32. Tan YS, Kim M, Kingsbury TJ, Civin CI, Cheng WC. Regulation of RAB5C is important for the growth inhibitory effects of MiR-509 in human precursor-B acute lymphoblastic leukemia. PloS one. 2014:9(11):e111777.

33. Onodera Y, Nam JM, Hashimoto A, Norman JC, Shirato H, Hashimoto S, et al. Rab5c promotes AMAP1-PRKD2 complex formation to enhance beta1 integrin recycling in EGF-induced cancer invasion. The Journal of cell biology. 2012;197(7):983-96

34. Wang Y, Jiang T. Understanding high grade glioma: molecular mechanism, therapy and comprehensive management. Cancer letters. 2013;331(2):139-46.

35. Lombardi D, Accorona R, Paderno A, Cappelli C, Nicolai P. Morbidity of central neck dissection for papillary thyroid cancer. Gland surgery. 2017;6(5):492-500.

36. Bagga S, Bracht J, Hunter S, Massirer K, Holtz J, Eachus R, et al. Regulation by let-7 and lin-4 miRNAs results in target mRNA degradation. Cell. 2005;122(4):553-63.

37. Zhao X, Zhang W, Ji W. MYO5A inhibition by miR-145 acts as a predictive marker of occult neck lymph node metastasis in human laryngeal squamous cell carcinoma. OncoTargets and therapy. 2018;11:3619-35 